\title{
Accuracy and Influencing factors of Pulmonary Artery Systolic Pressure estimated by Doppler Echocardiography: Comparsion with Right Heart Catheterization
}

\section{Guangjie Lv}

China-Japan Friendship Hospital https://orcid.org/0000-0001-8311-5353

Aili Li ( $\square$ echoaili@163.com )

China-Japan Friendship Hospital

\section{Xincao Tao}

China-Japan Friendship Hospital

\section{Yanan Zhai}

China-Japan Friendship Hospital

\section{Yu Zhang}

China-Japan Friendship Hospital

\section{Jieping Lei}

China-Japan Friendship Hospital

\section{Qian Gao}

China-Japan Friendship Hospital

\section{Wanmu Xie}

China-Japan Friendship Hospital

\section{Zhenguo Zhai}

China-Japan Friendship Hospital

\section{Research Article}

Keywords: Doppler echocardiography, Pulmonary hypertension, Right heart catheterization, Tricuspid regurgitation

Posted Date: August 31st, 2021

DOl: https://doi.org/10.21203/rs.3.rs-838208/v1

License: @ (i) This work is licensed under a Creative Commons Attribution 4.0 International License. Read Full License 


\section{Abstract \\ Purpose}

This study aimed to explore the accuracy and influencing factors of noninvasive assessment of pulmonary artery systolic pressure by Doppler echocardiography ( $\mathrm{SPAP}_{\mathrm{ECHO}}$ ) with right heart catheterization $(\mathrm{RHC})$ as reference.

\section{Methods}

A total of 218 highly suspected pulmonary hypertension $(\mathrm{PH})$ patients who underwent RHC and echocardiography within 7 days were included. The ratio of (sPAP $\left.\mathrm{ECHO}^{-\mathrm{SPAP}} \mathrm{RHC}_{\mathrm{R}}\right) / \mathrm{sPAP} \mathrm{RHC}_{\mathrm{R}}$ was calculated and divided into three groups, namely, the underestimation group, accurate group and overestimation group by $\pm 10 \%$ as the boundary. The correlation and consistency between tricuspid regurgitation (TR) derived parameters and RHC results were tested by Pearson and Bland-Altaman methods. With $\mathrm{mPAP} \geq 25 \mathrm{mmHg}$ measured by RHC as the standard diagnostic criteria of $\mathrm{PH}, \mathrm{ROC}$ curve was used to evaluate the diagnostic efficacy. The influencing factors of SPAP $\mathrm{ECHO}_{\mathrm{O}}$ were analyzed by ordered regression analysis.

\section{Results}

$\operatorname{SPAP}_{\mathrm{ECHO}}$ had the greatest correlation coefficient $(r=0.781, \mathrm{P}<0.001)$ and best diagnostic efficiency $(A U C=0.98)$ compared with other TR related methods. Bland-Altman analysis demonstrated the bias of $\mathrm{SPAP}_{\mathrm{ECHO}}$ (mean bias $=0.07 \mathrm{mmHg}, 95 \%$ limits of agreement: -32.08 to $+32.22 \mathrm{mmHg}$ ) was lower than that of TR-PG (mean bias $=5.87 \mathrm{mmHg}, 95 \%$ limits of agreement: -26.46 to $+38.21 \mathrm{mmHg}$ ). The results of ordered regression analysis showed that TR signal quality, PAWP and $\mathrm{SPAP}_{\mathrm{RHC}}$ level affected the accuracy of $\operatorname{SPAP}_{E C H O}(P<0.05)$. Relative to the signal quality of type $A$, the $O R$ value of type $B$ and $C$ signal quality were $0.26(95 \% \mathrm{Cl}: 0.14,0.48)$ and $0.23(95 \% \mathrm{Cl}: 0.07,0.73)$, respectively. The OR value of PAWP was $0.94(95 \% \mathrm{Cl}: 0.89,0.99)$. Compared with high $\mathrm{sPAP}_{\mathrm{RHC}}$ level, the OR value of low and medium sPAP $_{\mathrm{RHC}}$ level were $21.56(95 \% \mathrm{Cl}: 9.57,48.55)$ and $5.13(95 \% \mathrm{Cl}: 2.55,10.32)$, respectively.

\section{Conclusion}

$\mathrm{SPAP}_{\mathrm{ECHO}}$ was superior than other TR-related methods in screening PH. TR signal quality is vital for accurate assessment of $\mathrm{SPAP}_{\mathrm{ECHO}}$. For patients with pre-capillary disease, a higher echocardiographic threshold is more suitable for determining the possibility of $\mathrm{PH}$, especially during the initial stage of disease. 


\section{Introduction}

Noninvasive assessment of pulmonary artery systolic pressure by Doppler echocardiography (sPAP $\mathrm{ECHO}_{\text {) }}$ has been well adopted in pulmonary hypertension $(\mathrm{PH})$ screening and proved to be a reliable method ${ }^{[1]}$. Peak velocity of tricuspid regurgitation (TR Vmax) and its derived parameters including TR pressure gradient (TR-PG), TR mean pressure gradient (TR-mPG) and estimated mean pulmonary artery pressure

$\left(\mathrm{mPAP}_{\mathrm{ECHO}}\right)$ were also performed in clinical work, but whether $\mathrm{SPAP}_{\mathrm{ECHO}}$ is superior to other parameters in determining the probability of $\mathrm{PH}$ is worth further study. Besides, $\mathrm{SPAP}_{\mathrm{ECHO}}$ can also provide valuable information in evaluating treatment response and even predicting prognosis ${ }^{[2,3]}$. But high proportion of overestimation or underestimation of $\mathrm{SPAP}_{\mathrm{ECHO}}$ still remained ${ }^{[4]}$. As previous studies showed, TR severity, TR signal quality, the level of pulmonary artery systolic pressure and right ventricular function would affect the accuracy of $\mathrm{SPAP}_{\mathrm{ECHO}}{ }^{[5-7]}$. The influence of heart function on the accuracy of $\mathrm{SPAP}_{\mathrm{ECHO}}$ is unknown. In order to comprehensively evaluate $\mathrm{PH}$ patients' condition and improve doctor's diagnostic confidence, we need to understand in what kind of situation that $\mathrm{SPAP}_{\mathrm{ECHO}}$ will be under or overestimated. Therefore, the first aim of this study was to compare the efficiency of $\mathrm{SPAP}_{\mathrm{ECHO}}$ with other parameters in $\mathrm{PH}$ screening. And the second goal was to find influencing factors that account for the inaccuracy of $\mathrm{SPAP}_{\mathrm{ECHO}}$

\section{Methods}

From October 2015 and October 2020, a total of 430 patients with known or suspected PH admitted to our center were evaluated. Inclusion criteria included: age $\geq 18$ years old; the interval between echocardiography and RHC $\leq 7$ days; Exclusion criteria included: patients without TR, pulmonary artery stenosis or right ventricular outflow tract stenosis, poor image quality which are not suitable for analysis, ventricular septal defect or patent ductus arteriosus. Patient's demographic and clinical data were obtained from the departmental electronic medical record. The institutional review board of the ChinaJapan Friendship Hospital waived the need for written patient informed consent as this study involved the retrospective analysis of clinically acquired data. The data underlying this article will be shared on reasonable request to the corresponding author.

Baseline assessment of eligible patients including WHO functional class, the level of $\mathrm{N}$-terminal pro Btype natriuretic peptide (NT-proBNP) and 6-minute walk test (6MWT) were recorded.

\section{Right heart catheterization}

Haemodynamic measurements were performed with a 7F Swan-Ganz catheter Philips Allura X-PER FD20 flat-plate angiography system (Baxter Inc). The system was zeroed and referenced at patients' heart level as previously described ${ }^{[8]}$. Right atrial pressure (RAP), pulmonary systolic artery pressure $\left(\mathrm{SPAP}_{\mathrm{RHC}}\right)$ and pulmonary artery wedge pressure (PAWP) were recorded at end expiration in baseline over at least 3 heart cycles. Cardiac output (CO) was obtained using Fick's method. Pulmonary vascular resistance (PVR), 
cardiac index, stroke volume, pulse pressure and diastolic pressure gradient were calculated using standard formulas. According to the tertiles of $\mathrm{SPAP}_{\mathrm{RHC}}, \mathrm{PH}$ was classified into low, medium and high levels.

\section{Echocardiography}

Echocardiographic images were acquired using a GE Vivid E95 machine (GE Healthcare, General Electric Healthcare) equipped with M5S phased-array transducers. Analysis was performed independently by two blinded investigators using EchoPAC software (GE Healthcare version 201). Two-dimensional and Doppler echocardiography (DE) were performed on the basis of current guidelines. TR-PG was calculated from the TR Vmax obtained from continuous-wave Doppler by the simplified Bernoulli equation:TR-PG = 4 (TR Vmax $)^{2}$. TR-mPG was obtained by tracing the time-velocity of TR. SPAP $\mathrm{ECHO}_{\mathrm{O}}$ and $\mathrm{mPAP} \mathrm{ECHO}_{\mathrm{W}}$ were calculated by adding the estimated RAP to TR-PG and TR-mPG, respectively. RAP is divided into three categories $(3,8$, and $15 \mathrm{mmHg}$ ) based on the inferior vena cava (IVC) diameter and its respiratory variation ${ }^{[9]}$. Noninvasive assessment of $\mathrm{SPAP}_{\mathrm{ECHO}}$ was obtained by adding the right ventricle-right atrium pressure gradient (RV-RA PG) to the estimated RAP. The ratio of (sPAP $\left.\mathrm{ECHO}-\mathrm{SPAP}_{\mathrm{RHC}}\right) / \mathrm{sPAP} \mathrm{RHC}_{\mathrm{RH}}$ was calculated and divided into three groups, namely, the underestimation group, accurate group and overestimation group by $\pm 10 \%$ as the boundary. The severity of TR was classified into 3 grades by comprehensively evaluating the regurgitation jet area and vena contracta (VC) width. The mild group was defined as jet area $<5 \mathrm{~cm}^{2}$, VC TR $\leq 3 \mathrm{~mm}$; the moderate group as jet area $5-10 \mathrm{~cm}^{2}, 3 \mathrm{~mm}<\mathrm{VCTR} \nabla 7$ $\mathrm{mm}$ and severe group as jet area $>10 \mathrm{~cm}^{2}, V C T R \geq 7 \mathrm{~mm}$. The quality of the TR signal was classified into 3 types according to envelope visibility (Fig 1, type A, complete envelope; type B, partial envelope but prone to extrapolation; and type $C$, unreliable envelope) as previously reported ${ }^{[6]}$. RV systolic function was assessed using multiple parameters, including tricuspid annular plane systolic excursion (TAPSE), systolic annular tissue velocity of the lateral tricuspid annulus ( $\mathrm{S}^{\prime}$ ) and RV fractional area change (FAC). All parameters were repeatedly measured and averaged. To determine the reproducibility of SPAP $\mathrm{ECHO}_{\mathrm{O}}$ measurements, a total of 34 randomly selected examinations were analyzed twice by a first investigator at a 1-week interval and once by a second investigator.

\section{Statistical Analysis}

Standard statistical software (SPSS version 26 for Windows, SPSS, Chicago, IL, USA) was used for the statistical analysis. Data are expressed as mean \pm standard deviation for quantitative variables with normal distribution or as median (interquartile range) for variables without normal distribution. The correlation and consistency between TR derived parameters and RHC results were tested by Pearson and Bland-Altaman methods. With mPAP $\geq 25 \mathrm{mmHg}$ measured by RHC as the standard diagnostic criteria of $\mathrm{PH}, \mathrm{ROC}$ curve was used to evaluate the diagnostic efficacy. The influencing factors of $\mathrm{SPAP}_{\mathrm{ECHO}}$ were analyzed by ordered regression analysis. The intraclass correlation coefficient was used to determine inter- and intra-observer reproducibility for $\mathrm{SPAP}_{\mathrm{ECHO}}$ from 34 randomly selected patients using an 
identical cine-loop for each view. For all statistical tests, a $P$ value $<0.05$ was used to indicate significance.

\section{Results}

\section{Patients characteristics}

A total of 218 patients were finally identified and analyzed, as shown in Fig 2. Baseline demographics and clinical characteristics are described in Table 1. The mean age of patients was $50.9 \pm 13.3$ years old, 40.3\% were male, 197 (90.4\%) patients had PH. No patient experienced major cardiac event between DE and RHC examinations. Table 2 lists the DE and RHC variables grouped by estimated accuracy.

\section{Observer variability of $\mathrm{SPAP}_{\mathrm{ECHO}}$ Estimation}

The intraclass correlation coefficient for inter-observer reproducibility of SPAP $\mathrm{ECHO}_{\mathrm{O}}$ was 0.988 (95\% confidence interval 0.977-0.994) and the intraclass correlation coefficient for intra-observer reproducibility of $\mathrm{SPAP}_{\mathrm{ECHO}}$ was 0.992 (95\% confidence interval $\left.0.984-0.996\right)$.

\section{Association between invasively determined parameters and TR derived parameters}

All the TR derived parameters including TR Vmax, TR-PG, TR-mPG, mPAP $\mathrm{ECHO}_{\mathrm{O}}$ and sPAP $\mathrm{ECHO}_{\mathrm{O}}$ showed positive correlation with related $\mathrm{RHC}$ results (Fig 3). $\mathrm{SPAP}_{\mathrm{ECHO}}$ had the greatest correlation coefficient $(r=0.782, \mathrm{P}<0.001)$. Bland-Altman analysis demonstrated low bias between RHC and echocardiographic results with wide limits of agreements (Fig 4). The bias of $\mathrm{SPAP}_{\mathrm{ECHO}}$ (mean bias $=0.07 \mathrm{mmHg}, 95 \%$ limits of agreement: -32.08 to $+32.22 \mathrm{mmHg}$ ) was lower than that of TR-PG (mean bias $=5.87 \mathrm{mmHg}, 95 \%$ limits of agreement: -26.46 to $+38.21 \mathrm{mmHg}$ ). The mean deviation between $\mathrm{MPAP}_{\mathrm{ECHO}}$, TR-mPG with mPAP $\mathrm{RHC}$ were $-2.59 \mathrm{mmHg}(95 \%$ limits of agreement -26.29 to $+21.11 \mathrm{mmHg}$ ) and $3.32 \mathrm{mmHg}$ ( $95 \%$ limits of agreement -20.07 to $+26.70 \mathrm{mmHg}$ ), respectively.

\section{Performance of different TR methods for predicting PH}

TR Vmax, TR-PG, TR-mPG, mPAP $\mathrm{ECHO}_{\mathrm{O}}$ and $\mathrm{SPAP}_{\mathrm{ECHO}}$ showed similar diagnostic performance for determining the possibility of $\mathrm{PH}$ (Table 3 ). There was no significant difference among the AUCs of these TR methods in predicting $\mathrm{PH}(P \otimes 0.05)$. However, the predictive efficiency and sensitivity of $\mathrm{SPAP}_{\mathrm{ECHO}}$ were better than other methods.

\section{Factors affecting the accuracy of $\mathrm{SPAP}_{\mathrm{ECHO}}$ estimation}

There were 79 patients $(36.2 \%)$ in overestimated group, 81 patients $(37.2 \%)$ in accurate group and 58 patients $(26.6 \%)$ in underestimated group. $\mathrm{SPAP}_{\mathrm{RHC}}$ was divided into three levels according to its tertile $(63 \mathrm{mmHg}, 85 \mathrm{mmHg})$. Univariable ordered analysis demonstrated that RV WT, FAC, TR signal quality, SPAP $_{\mathrm{RHC}}$ level, RAP, PVR, PAWP and mPAP were associated with inaccuracy of $\mathrm{SPAP}_{\mathrm{ECHO}}$ estimation 
(Table 2). After multivariate ordered regression analysis, there were significant differences on TR signal quality, PAWP and $\mathrm{SPAP}_{\mathrm{RHC}}$ level $(\mathrm{P}<0.05)$. Relative to the signal quality of type $A$, the $O R$ value of type $B$ and $C$ signal quality were $0.26(95 \% \mathrm{Cl}: 0.14,0.48)$ and $0.23(95 \% \mathrm{Cl}: 0.07,0.73)$, respectively. The OR value of PAWP was $0.94(95 \% \mathrm{Cl}: 0.89,0.99)$. Compared with high $\mathrm{sPAP}_{\mathrm{RHC}}$ level, the OR value of low and medium SPAP ${ }_{\mathrm{RHC}}$ level were $21.56(95 \% \mathrm{Cl}: 9.57,48.55)$ and $5.13(95 \% \mathrm{Cl}: 2.55,10.32)$, respectively.

\section{Discussion}

Key findings of our study: (1) Compared with TR Vmax and its derived parameters, sPAP $\mathrm{ECHO}_{\text {showed }}$ better sensitivity for predicting PH while maintaining similar specificity. (2) The accuracy of SPAP $_{\mathrm{ECHO}}$ could be affected by TR signal quality, PAWP and $\mathrm{SPAP}_{\mathrm{RHC}}$ level.

\section{Performance of $\mathrm{SPAP}_{\mathrm{ECHO}}$ in $\mathrm{PH}$ screening}

In our study, $\mathrm{SPAP}_{\mathrm{ECHO}}$ exhibited best correlation with $\mathrm{SPAP}_{\mathrm{RHC}}$ and was superior to TR Vmax and its derived methods in $\mathrm{PH}$ screening. $\mathrm{SPAP}_{\mathrm{ECHO}}$ didn't amplify measurement errors in assessing pulmonary artery pressure as a derived variables of TR Vmax as mentioned in the currents guideline ${ }^{[9]}$, on the contrary, it showed better sensitivity while maintaining similar specificity. Moreover, $\mathrm{SPAP}_{\mathrm{ECHO}}$ and $\mathrm{mPAP}_{\mathrm{ECHO}}$ contain more information from RAP relative to TR-PG and TR-mPG which may accounts for their better accuracy and lower bias. In this cohort, the RAP was appropriately evaluated in $66.2 \%$ of cases (defined as RAP estimated by echocardiography was in the same range of RAP measured by RHC) , $13.9 \%$ were underestimated and $19.9 \%$ were overestimated. In addition, $76.2 \%(16 / 25)$ of cases whose RAP was overestimated make up for the underestimation of $\mathrm{SPAP}_{\mathrm{ECHO}}$ to varying degrees. $\ln 72.2 \%$

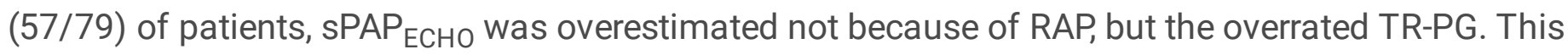
phenomenon demonstrated that RAP estimated by echocardiography is reliable and could add additional

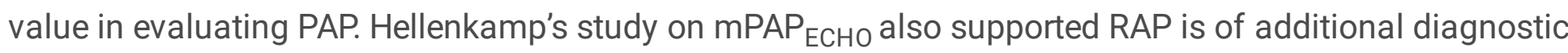
value in predicting $\mathrm{PH}^{[10]}$. But Venkateshvaran's research suggested recommendation based RAP demonstrated poor precision and may not necessarily contribute to greater accuracy of pulmonary artery pressure estimates ${ }^{[11]}$. Until now, there is no consensus on the role of RAP, but we can't deny that elevated RAP is an important sign for $\mathrm{PH}$. So further studies are needed to figure out those uncertainties and make RAP a more valuable parameter to evaluate $\mathrm{PH}$.

\section{Reasons for inaccuracy of sPAP $\mathrm{ECHO}_{\mathrm{O}}$ estimation}

First, our finding confirmed previous reports that the TR signal quality is vital for accurate assessment of $\mathrm{SPAP}_{\mathrm{ECHO}}{ }^{[6]}$. But previous studies also reported the accuracy of SPAP $\mathrm{ECHO}_{\mathrm{O}}$ would be affected by TR severity ${ }^{[5,12]}$. So the relationship between TR severity and TR signal quality was further analyzed. In our cohort, the difference in TR signal quality between different TR severity was statistically significant $(P]$ 0.05). For patients with severe TR, $81.2 \%$ of them had type $A$ signal quality and $S P A P_{E C H O}$ were evaluated appropriately in $61.9 \%$ of them. While for patients with mild or moderate TR, $54.8 \%$ of them obtained type 
A signal quality and $37.1 \%$ of them estimated $\mathrm{SPAP}_{\mathrm{ECHO}}$ accurately. In this study, patients with severe TR seem to have a better chance to get complete signal quality and assess SPAP $\mathrm{ECHO}_{\mathrm{O}}$ properly. But TR severity didn't include in the final equation. The possible explanations might be what truly determined the accuracy of $\mathrm{SPAP}_{\mathrm{ECHO}}$ assessment is TR signal quality. Even with mild or moderate TR, as long as signal envelope is complete, accurate assessment could be achieved. Moreover, $9.6 \%$ of patients in our cohort had severe TR which is in line with the actual clinical situation that severe TR only appears in the minority of the total patients. On the whole, the impact of TR severity on the accuracy of $\mathrm{SPAP}_{\mathrm{ECHO}}$ evaluation is not as significant as TR signal quality.

Thus, in order to avoid underestimation of $\mathrm{SPAP}_{\mathrm{RHC}}$ in clinical practice, it is necessary to perform multisection and multi-angle measurement of echocardiographic images to obtain a more complete signal envelope, especially for patients with mild TR. And for patients without TR, mPAP estimated by pulmonary regurgitation is also a useful alternative for screening $\mathrm{PH}$.

Second, our data also suggested patients with higher PAWP tended to underestimate SPAP $_{\mathrm{RHC}}$ by $\mathrm{DE}$. Finkelhor et al also found PAWP had a strong inverse correlation with the difference between $\mathrm{SPAP}_{\mathrm{RHC}}$ and $\mathrm{SPAP}_{\mathrm{ECHO}}{ }^{[13]}$. They speculated that elevated left atrial pressure can be transmitted to the right atrium via the shared inter-atrial septum as well as through pericardial constraint and limit TR velocities, thus the accuracy of $\mathrm{SPAP}_{\mathrm{ECHO}}$ will be affected. Even though the mechanism of how PAWP affects pulmonary artery pressure is still unclear, interestingly, more and more studies are beginning to notice this phenomenon. Amsallem et al found patients with higher PAWP is assosiated with lower $\mathrm{SPAP}_{\mathrm{ECHO}}$ thresholds for $\mathrm{PH}$ diagnosis ${ }^{[14]}$. Our results also supported this view, the optimal cut-off value of our cohort for determining $\mathrm{PH}$ was $55 \mathrm{mmHg}$, which is much higher than previous studies. Patients with pre-capillary $\mathrm{PH}(85.8 \%)$ accounted for the majority of our population may explain this discrepancy. Patients with left heart diseases (post-capillary) PH took up for $84 \%$ and $76 \%$ in these two researches, respectively ${ }^{[1,15]}$. This finding suggests that the best threshold of pre-capillary $\mathrm{PH}$ might be higher than post-capillary PH and PAWP may play an important role in it. Therefore, a higher threshold of $\mathrm{SPAP}_{\mathrm{ECHO}}$ for determining pre-capillary $\mathrm{PH}$ is more appropriate.

Third, for the effect of $\mathrm{SPAP}_{\mathrm{RHC}}$ level on the accuracy of pulmonary artery pressure estimated by TR, Groh et al found DE inaccurately estimated right ventricular pressure in children with elevated right heart pressure ${ }^{[16]}$. Our results provided further evidence that $D E$ tended to overestimate SPAP $_{\mathrm{RHC}}$ at low $\mathrm{SPAP}_{\mathrm{RHC}}$ level and increasingly underestimated the $\mathrm{sPAP}_{\mathrm{RHC}}$ with the advance of $\mathrm{sPAP}_{\mathrm{RHC}}$ level. We speculated that the coupling mechanism between RV contractility and its load may account for this phenomenon. $\mathrm{sPAP}_{\mathrm{RHC}}$ is mildly elevated during the initial phase of $\mathrm{PH}, \mathrm{RV}$ coupling is maintained by a 4to 5 -fold increase in contractility through muscle hypertrophy as well as changes in muscle properties ${ }^{[17]}$. The compensatory enhancement of RV contractility ${ }^{[18]}$ would make TR Vmax become higher, sPAP $_{\mathrm{RHC}}$ will be overestimated by $\mathrm{DE}$, while the PAP is still in the normal range due to the natural vascular elasticity. As SPAP ${ }_{\mathrm{RHC}}$ increased moderately, the compensatory contractility of RV would halt and the 
stroke volume (SV) would decrease, but $\mathrm{CO}$ is maintained by increasing heart rate. At this stage of $\mathrm{PH}$, the estimation of $\mathrm{SPAP}_{\mathrm{RHC}}$ by $\mathrm{DE}$ is relatively reliable. However, $\mathrm{SPAP}_{\mathrm{RHC}}$ would become higher with the development of $\mathrm{PH}$, when $\mathrm{RV}$ uncoupling occurs, $\mathrm{CO}$ would reduce which will result in increasing of RV preload. The elevated RV preload and RAP would lead to a decreased right atrial-ventricular pressure gradience, thus DE would underestimate the $\mathrm{SPAP}_{\mathrm{RHC}}$. $\mathrm{SPAP}_{\mathrm{RHC}}$ level may affect the accuracy of $\mathrm{SPAP}_{\mathrm{ECHO}}$ through coupling mechanism between RV contractility and its load, but studies with larger sample sizes are needed test this hypothesis.

As our result indicated that $\mathrm{DE}$ tends to overestimate the pressure at low $\mathrm{SPAP}_{\mathrm{RHC}}$ level. So a diagnostic threshold higher than the empirical formula is more suitable for assigning the likelihood of PH during the initial stage of disease to avoid excessive invasive examination. For patients with high $\mathrm{sPAP}_{\mathrm{RHC}}$ level, attention should be paid to comprehensively assess RV contractility, TR severity and other signs to avoid underestimation of pressure and wrong evaluation of condition. At last, we didn't find RV systolic parameters and heart function index (6M WT and WHO functional class) have significant impact on the accuracy of $\mathrm{SPAP}_{\mathrm{ECHO}}$ neither. Theoretically, RV systolic function will gradually decrease ${ }^{[19]}$, but the RV can remain coupled for the large increase in load by increasing contractility until heart failure ${ }^{[17]}$. Therefore, RV systolic parameters are relatively stable before the end stage of $\mathrm{PH}$. In addition, the heart movement and measurement angle dependence also affect the accuracy of the relevant parameters. While the evaluation of heart function index is closely related to the coordination of patients and the subjectivity of physician which may bring uncertainties. Although RV systolic parameters and heart function index had clinical significance for the assessment of $\mathrm{PH}$, they didn't have significant effect on the accuracy of $\mathrm{SPAP}_{\mathrm{ECHO}}$ estimation.

\section{Limitations}

This study has several limitations that merits emphasis. First, this is a retrospective research with a small sample size. $90.5 \%$ had $\mathrm{PH}$ and $47.7 \%$ of them were due to chronic pulmonary thromboembolism, the sample size of other type PH was relatively small. Thus we couldn't give specific suggestion for each type of $\mathrm{PH}$. Further more, interval time between $\mathrm{RHC}$ and echocardiography was within 7 days which is longer than previous reports. But the average interval time was 3 days in this study, and the majority of our patients have pre-capillary PH which indicates the patient's hemodynamics are relatively stable and wouldn't change dramatically during this short time. At last, the single-center nature of the present study limited generalization. The majority of our patients were pre-capillary $\mathrm{PH}$, therefore, the conclusion is mainly applicable to patients with precapillary $\mathrm{PH}$.

\section{Conclusion}

SPAP $_{\mathrm{ECHO}}$ was superior than other TR-related methods in screening PH. TR signal quality is vital for accurate assessment of $\mathrm{SPAP}_{\mathrm{ECHO}}$. For patients with pre-capillary disease, a higher echocardiographic 
threshold is more suitable for determining the possibility of $\mathrm{PH}$, especially during the initial stage of disease.

\section{References}

1. Greiner S, Jud A, Aurich M et al (2014) Reliability of noninvasive assessment of systolic pulmonary artery pressure by Doppler echocardiography compared to right heart catheterization: analysis in a large patient population[J]. J Am Heart Assoc 3(4):e1103

2. Santiago-Vacas E, Lupon J, Gavidia-Bovadilla G et al (2020) Pulmonary hypertension and right ventricular dysfunction in heart failure: prognosis and 15-year prospective longitudinal trajectories in survivors[J]. Eur J Heart Fail 22(7):1214-1225

3. Jalalian R, Moghadamnia AA, Tamaddoni A et al (2017) Comparing the Efficacy of Tadalafil Versus Placebo on Pulmonary Artery Systolic Pressure and Right Ventricular Function in Patients with BetaThalassaemia Intermedia[J]. Heart Lung Circ 26(7):677-683

4. Li Y, Wang Y, Li H et al (2017) Evaluation of the hemodynamics and right ventricular function in pulmonary hypertension by echocardiography compared with right-sided heart catheterization[J]. Exp Ther Med 14(4):3616-3622

5. Fei B, Fan T, Zhao L et al (2017) Impact of severe tricuspid regurgitation on accuracy of systolic pulmonary arterial pressure measured by Doppler echocardiography: Analysis in an unselected patient population[J]. Echocardiography 34(7):1082-1088

6. Amsallem M, Sternbach JM, Adigopula S et al (2016) Addressing the Controversy of Estimating Pulmonary Arterial Pressure by Echocardiography[J]. J Am Soc Echocardiogr 29(2):93-102

7. Gual-Capllonch F, Lupon J, Bancu I et al (2021) Preload dependence of pulmonary haemodynamics and right ventricular performance[J]. Clin Res Cardiol 110(4):591-600

8. Krishnan A, Markham R, Savage M et al (2019) Right Heart Catheterisation: How To Do It[J]. Heart Lung Circ 28(4):e71-e78

9. Galie N, Humbert M, Vachiery JL et al (2015) 2015 ESC/ERS Guidelines for the diagnosis and treatment of pulmonary hypertension: The Joint Task Force for the Diagnosis and Treatment of Pulmonary Hypertension of the European Society of Cardiology (ESC) and the European Respiratory Society (ERS): Endorsed by: Association for European Paediatric and Congenital Cardiology (AEPC), International Society for Heart and Lung Transplantation (ISHLT)[J]. Eur Respir J 46(4):903-975

10. Hellenkamp K, Unsold B, Mushemi-Blake S et al (2018) Echocardiographic Estimation of Mean Pulmonary Artery Pressure: A Comparison of Different Approaches to Assign the Likelihood of Pulmonary Hypertension[J]. J Am Soc Echocardiogr 31(1):89-98

11. Venkateshvaran A, Seidova N, Tureli HO et al. Accuracy of echocardiographic estimates of pulmonary artery pressures in pulmonary hypertension: insights from the KARUM hemodynamic database[J]. Int J Cardiovasc Imaging, 2021. https://doi.org/ 10.1007/s10554-021-02315-y 
12. Hioka T, Kaga S, Mikami T et al (2017) Overestimation by echocardiography of the peak systolic pressure gradient between the right ventricle and right atrium due to tricuspid regurgitation and the usefulness of the early diastolic transpulmonary valve pressure gradient for estimating pulmonary artery pressure[J]. Heart Vessels 32(7):833-842

13. Finkelhor RS, Scrocco JD, Madmani M et al (2014) Discordant Doppler right heart catheterization pulmonary artery systolic pressures: importance of pulmonary capillary wedge pressure[J]. Echocardiography 31(3):279-284

14. Amsallem M, Tedford RJ, Denault A et al (2020) Quantifying the Influence of Wedge Pressure, Age, and Heart Rate on the Systolic Thresholds for Detection of Pulmonary Hypertension[J]. J Am Heart Assoc 9(11):e16265

15. Sawada N, Kawata T, Daimon M et al (2019) Detection of Pulmonary Hypertension with Systolic Pressure Estimated by Doppler Echocardiography[J]. Int Heart J 60(4):836-844

16. Groh GK, Levy PT, Holland MR et al (2014) Doppler echocardiography inaccurately estimates right ventricular pressure in children with elevated right heart pressure[J]. J Am Soc Echocardiogr 27(2):163-171

17. Vonk NA, Westerhof BE, Westerhof N (2017) The Relationship Between the Right Ventricle and its Load in Pulmonary Hypertension[J]. J Am Coll Cardiol 69(2):236-243

18. Margonato D, Ancona F, Ingallina G et al (2021) Tricuspid Regurgitation in Left Ventricular Systolic Dysfunction: Marker or Target?[J]. Front Cardiovasc Med 8:702589

19. Kong D, Shu X, Pan C et al (2012) Evaluation of right ventricular regional volume and systolic function in patients with pulmonary arterial hypertension using three-dimensional echocardiography[J]. Echocardiography 29(6):706-712

\section{Tables}

Table 1 Clinical and demographic characteristics 


\begin{tabular}{|c|c|}
\hline Variables & Value \\
\hline Age (years) & $50.9 \pm 13.3$ \\
\hline Males $₫ \%$ & $90 \rrbracket 41.3 \rrbracket$ \\
\hline BMI & $\begin{array}{l}1.67 \rrbracket 1.57 \rrbracket \\
1.84 \rrbracket\end{array}$ \\
\hline Systolic BP(mmHg) & $120 \rrbracket 108 \rrbracket 132 \rrbracket$ \\
\hline Diastolic BP(mmHg) & $77 \rrbracket 70 \rrbracket 87 \rrbracket$ \\
\hline Heart rate (bpm) & $76 \llbracket 68.65 \rrbracket 80 \rrbracket$ \\
\hline Interval between TTE and RHC, days & $2.5 \rrbracket 1 \rrbracket 5 \rrbracket$ \\
\hline NT-pro BNP (pg/ml) & $451(175,1043 \rrbracket$ \\
\hline 6M WT (m) & $365.5 \pm 104.6$ \\
\hline \multicolumn{2}{|l|}{ WHO functional class } \\
\hline I Class $₫ \% \bigotimes$ & $20 \rrbracket 9.2 \rrbracket$ \\
\hline II Class $₫ \% \bigotimes$ & $93 \rrbracket 42.7 \rrbracket$ \\
\hline III Class $₫ \% \rrbracket$ & $89 ه 40.8 \rrbracket$ \\
\hline IV Class $\% \rrbracket$ & 16冈7.3囚 \\
\hline $\mathrm{PH}(\mathrm{n})$ & $197(90.4 \%)$ \\
\hline Idiopathic, heritable, drug and toxic induced & 37 \\
\hline Associated with Connective tissue disease & 25 \\
\hline Portal hypertension & 2 \\
\hline Congenital heart disease & 8 \\
\hline $\mathrm{PH}$ due to left heart disease & 6 \\
\hline $\mathrm{PH}$ due to lung disease and/or hypoxia & 6 \\
\hline Chronic thromboembolic $\mathrm{PH}$ & 95 \\
\hline $\mathrm{PH}$ with unclear and/or multifactorial mechanisms & 13 \\
\hline $\begin{array}{l}\text { Pulmonary veno-occlusive disease and/or pulmonary capillary } \\
\text { haemangiomatosis }\end{array}$ & 5 \\
\hline Non-PH (n) & $21(9.6 \%)$ \\
\hline
\end{tabular}

Values are presented as mean $\pm S D$, median (IQR), or $n(\%)$. 
$\mathrm{BMI}$, body mass index; TTE, transthoracic echocardiography; RHC, right heart catheterization; $\mathrm{BP}$, blood pressure; NT-pro BNP, N-terminal pro B-type natriuretic peptide; 6M WT, 6-minutes walk test; PH, pulmonary hypertension.

Table 3 Receiver Operating Characteristic Curve Analysis of DE Parameters for Detecting PH (mPAP $\geq$ $25 \mathrm{mmHg}$ )

\begin{tabular}{|lllllll|}
\hline & AUC & cutoff value & sensitivity (\%) & specificity (\%) & PPV(\%) & NPV(\%) \\
\hline SPAP $_{\text {ECHO }}$ & 0.980 & $55 \mathrm{mmHg}$ & 90.4 & 100 & 100 & 53 \\
\hline TR Vmax & 0.976 & $361 \mathrm{~cm} / \mathrm{s}$ & 88.8 & 100 & 100 & 49 \\
\hline TR-PG & 0.977 & $52.67 \mathrm{mmHg}$ & 87.8 & 100 & 100 & 47 \\
\hline mPAP & 0.956 & $30.42 \mathrm{mmHg}$ & 94.4 & 84.6 & 99 & 52 \\
\hline TR-mPG & 0.945 & $27.42 \mathrm{mmHg}$ & 92.7 & 84.6 & 99 & 50 \\
\hline
\end{tabular}

PPV: Positive predictive value; NPV: Negative predictive value. SPAP $_{\mathrm{ECHO}}$ : pulmonary systolic pressure estimated by echocardiography; TR Vmax囚maximum velocity of tricuspid regurgitation; TR-PG: tricuspid regurgitation pressure gradient; $\mathrm{MPAP}_{\mathrm{ECHO}}$ : mean pulmonary artery pressure estimated by echocardiography; TR-mPG: tricuspid regurgitation mean pressure gradient.

Due to technical limitations, Table 2 is only available as a download in the Supplemental Files section.

\section{Figures}

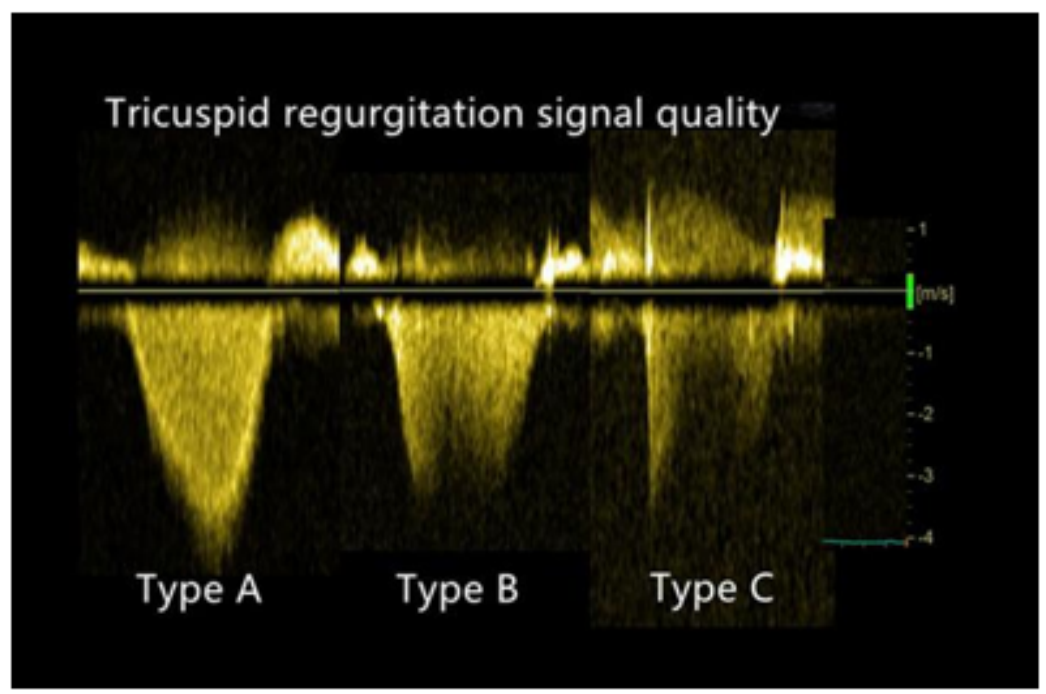

Figure 1 
Classification of the TR signal quality using continuous-wave Doppler. Type A, complete envelope; Type B, partial envelope but prone to extrapolation; Type C, unreliable envelope or no signal.

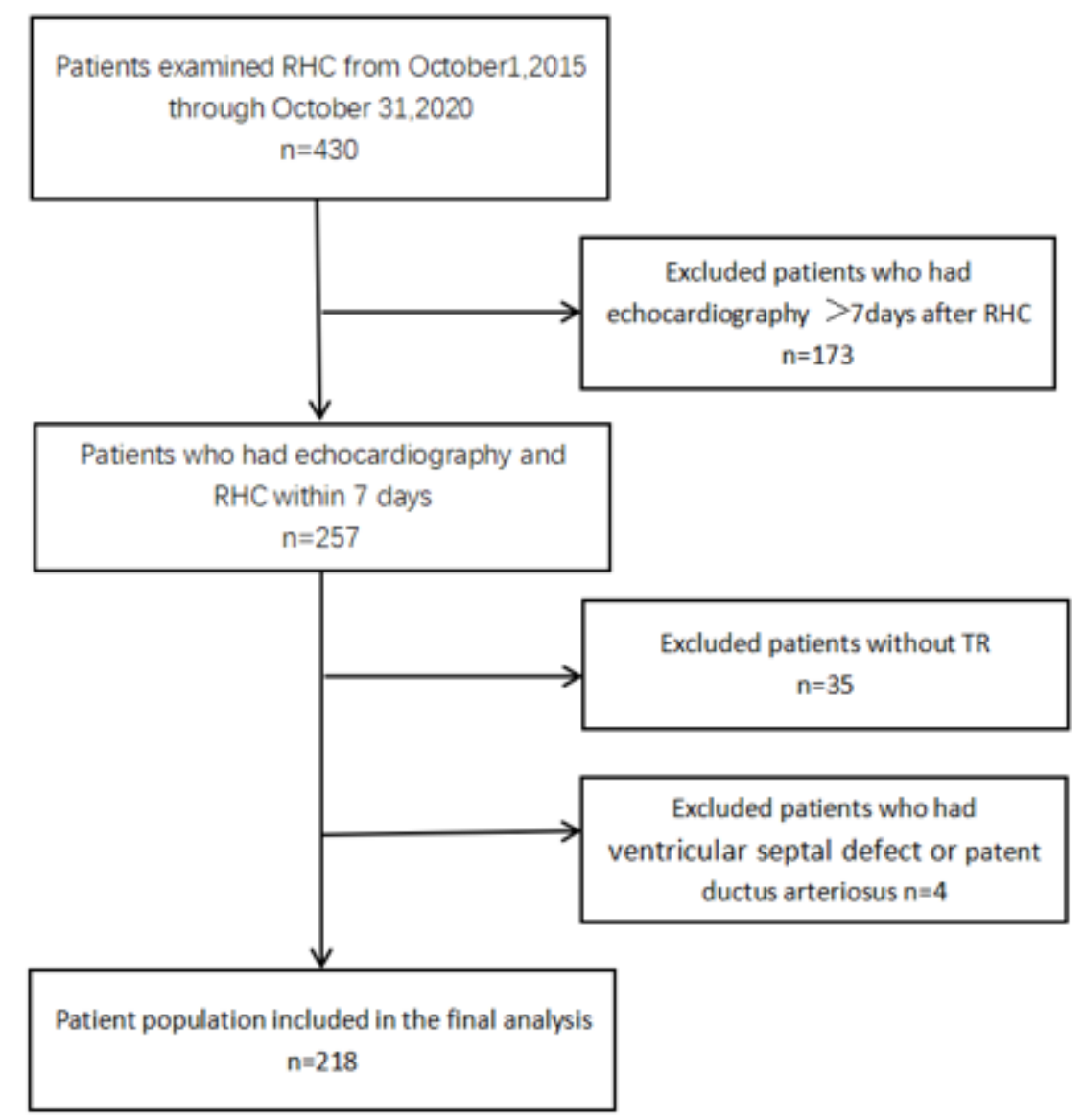

Figure 2

Flow chart of patient screening 

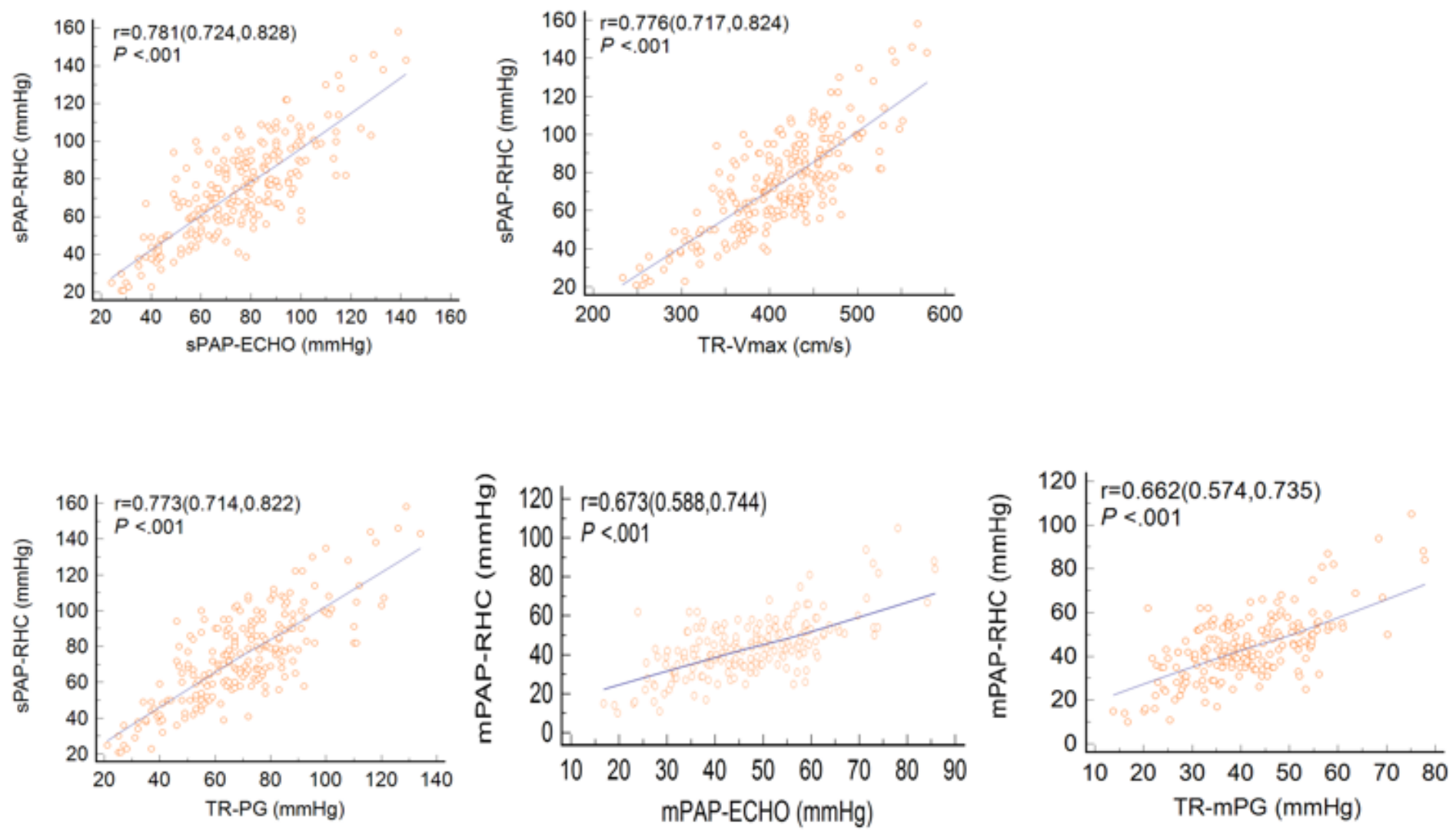

Figure 3

Correlation of invasively determined parameters with TR derived parameters. Spearman's rank correlation coefficients are presented with $95 \%$ Cls in brackets. 

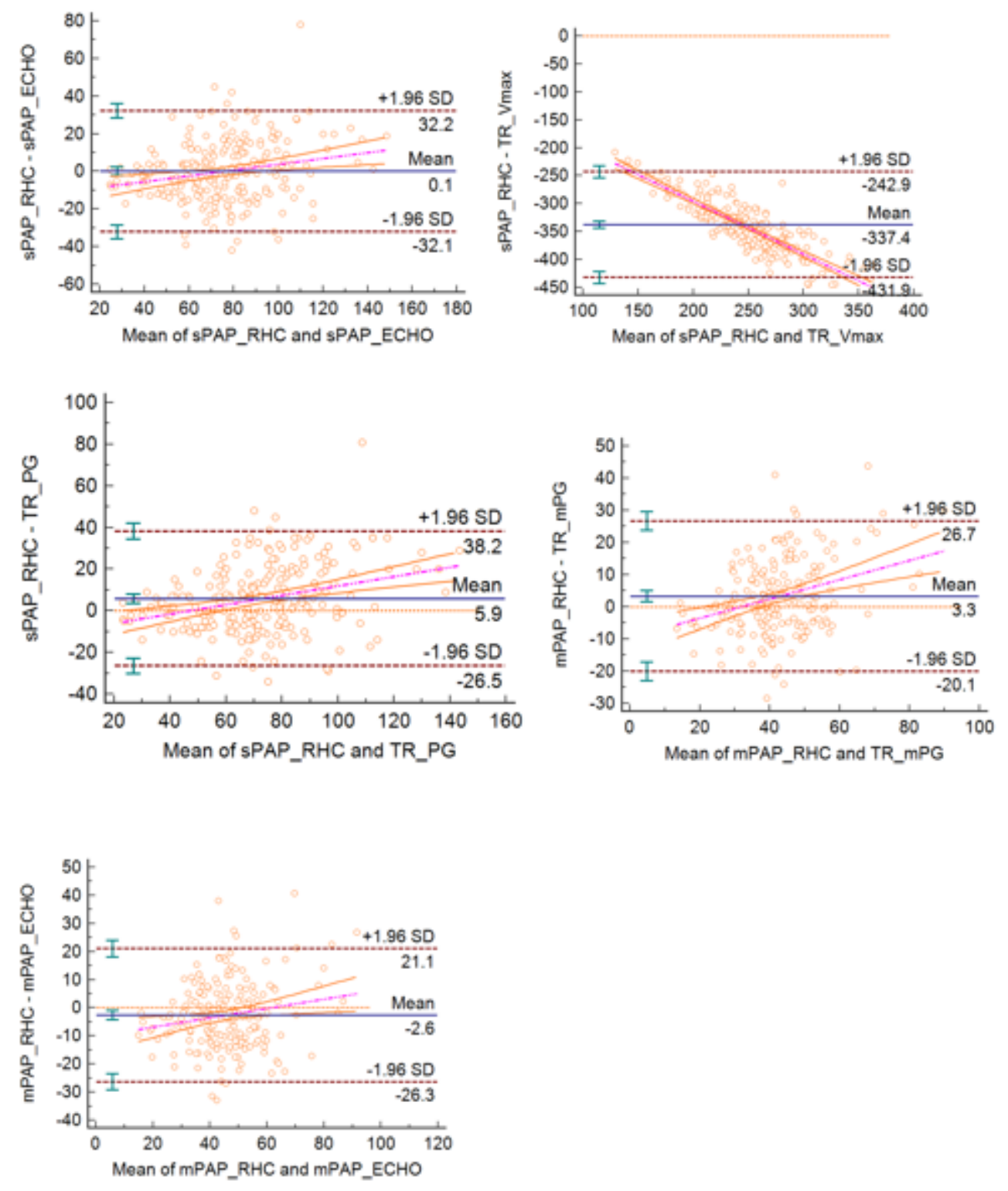

\section{Figure 4}

Bland-Altman plot showing the relationship between invasively determined parameters with TR derived parameters. 


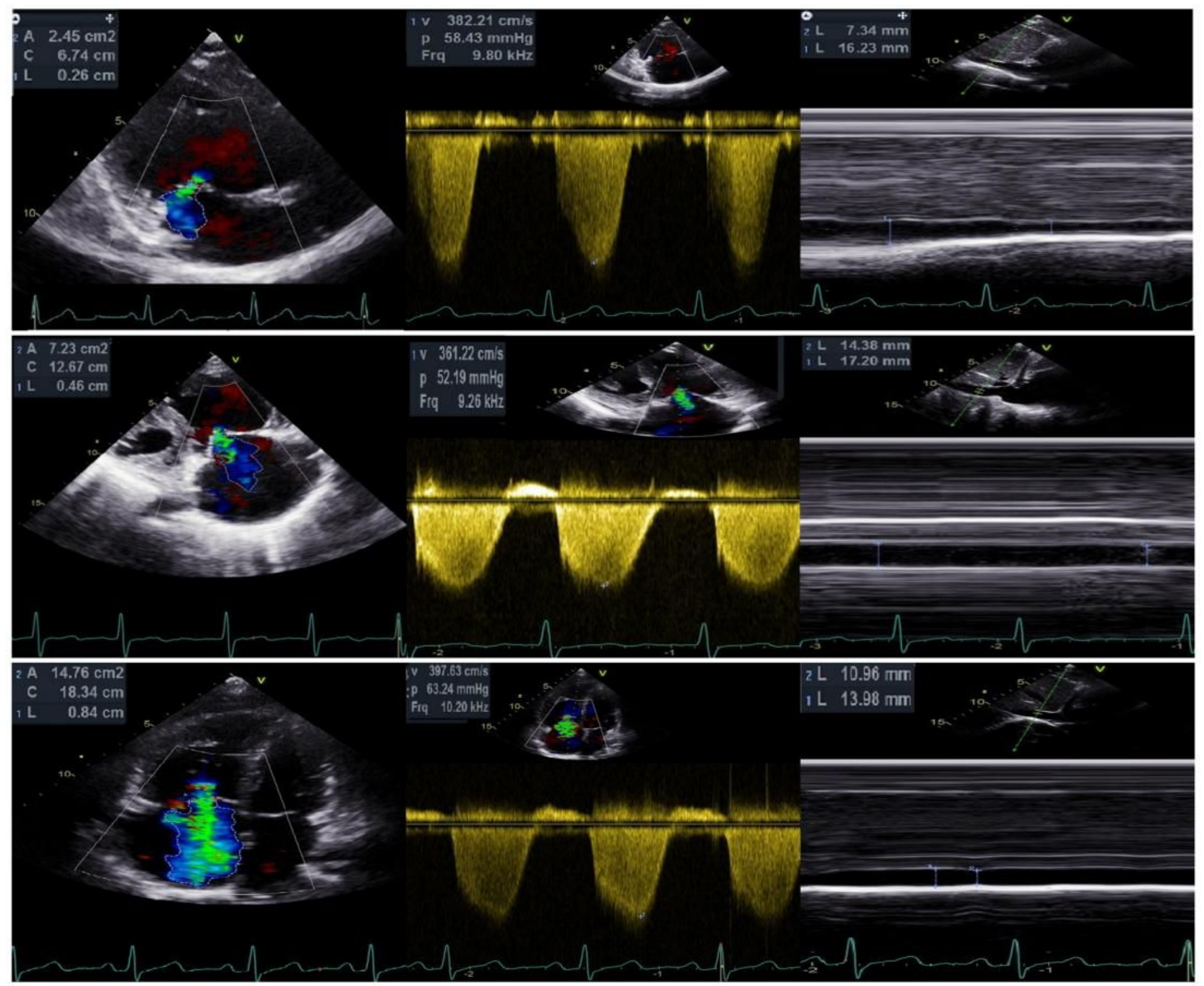

\section{Figure 5}

Example of different severity of TR with type A signal quality and accurate sPAPECHO. The upper image presents a 40 years old female with mild TR whose sPAPECHO and sPAPRHC were 59 and $61 \mathrm{mmHg}$, respectively. The medium image shows a 50 years old female with moderate TR whose sPAPECHO and SPAPRHC were 60 and $60 \mathrm{mmHg}$, respectively. The lower image demonstrates a 34 years old female with severe TR whose sPAPECHO and sPAPRHC were 71 and $73 \mathrm{mmHg}$, respectively.

\section{Supplementary Files}

This is a list of supplementary files associated with this preprint. Click to download.

- Table2UnivariableandmultivariableorderedanalysisforaccuracyofsPAPECHO.docx 